\title{
Nonsurgical management of gastroduodenal tuberculosis: Nine-year experience from a tertiary referral center
}

\section{(ㄷ)(1) $(9)$}

\author{
Authors \\ Ashok Dalal ${ }^{1}$, Amarender Singh Puri ${ }^{1}$, Sanjeev Sachdeva ${ }^{1}$, Puja Sakuja ${ }^{2}$ \\ Institutions \\ 1 Department of Gastroenterology, GB Pant Hospital, New \\ Delhi, India \\ 2 Department of Pathology, G B Pant Hospital, New Delhi, \\ India
}

submitted 6.6.2018

accepted after revision $\quad 15.10 .2018$

\section{Bibliography}

DOI https://doi.org/10.1055/a-0957-2754 |

Endoscopy International Open 2019; 07: E1248-E1252

(c) Georg Thieme Verlag KG Stuttgart · New York elSSN 2196-9736

Corresponding author

Dr. Amarender Singh Puri, Professor, Department of Gastroenterology, Academic Block, GB Pant Hospital, 1 JLN Marg, New Delhi, India 110002

Fax: +91-11- 23239442

amarender.puri@gmail.com

\section{ABSTRACT}

Background and study aims Gastroduodenal tuberculosis (GDTB) is an uncommon disease. Surgery has been standard of care both for diagnosis and management of GDTB. The aim of this study was to evaluate the efficacy of non-surgical management of GDTB using a combination of anti-tuberculous therapy (ATT) along with endoscopic dilatation of the tuberculous stricture.

Patients and methods Patients suspected to have gastroduodenal TB were evaluated: clinical, endoscopic, radiological, and histopathological data were recorded. Patients in whom a definite diagnosis of tuberculosis could not be confirmed on mucosal biopsies underwent endoscopic mucosal resection (EMR). Patients were treated with ATT and endoscopic dilatation was done if indicated. Patients were followed up to evaluate clinical, radiological and endoscopic response.

Results Over a 9-year period from 2009 to 2017, 52 patients (mean age 28.5yrs) were diagnosed with GDTB. The most common presenting symptoms were vomiting $(n=51$, $98 \%)$ and weight loss $(n=52,100 \%)$. The most common anatomical site of involvement was D1-D2 junction $(n=22$, $42 \%)$. Histopathological diagnosis could be made in 43 patients (82.6\%); 36 (69\%) on mucosal biopsies and in 7 of 10 patients $(70 \%)$ who underwent snare biopsy/EMR. Endoscopic dilatation was done in 37 patients (71\%) and median dilatation sessions were two. Failure of endotherapy occurred in four patients $(7.6 \%)$. All responders had complete amelioration of symptoms after 4 to 6 weeks of combination therapy. Median period of follow-up was 23.5 months and none of the patients reported any recurrence of symptoms. Conclusion ATT and endoscopic dilatation combined has a high success rate in management of GDTB and should be considered the standard of care.

\section{Introduction}

Tuberculosis (TB) of the gastrointestinal tract (GIT) accounts for $11 \%$ of extrapulmonary tuberculosis (EPTB) cases [1]. The ileocecal region is the most common site, accounting for more than $50 \%$ of GIT TB cases [2]. Gastric and duodenal involvement is rare in EPTB and is seen in only $1 \%$ of cases of GIT TB $[2,3]$. Low gastric $\mathrm{pH}$ due to gastric acid is thought to inhibit proliferation of Mycobacterium tuberculosis in the stomach [4]. The typical presenting symptom of GDTB is gastric outlet obstruction; in rare cases, patients may present with upper gastrointestinal bleeding or a mass lesion mimicking malignancy [5].

Prior to 2000, most cases of GDTB were anecdotal reports in which radiology was the cornerstone for the diagnosis [6-8]. In the first series of GDTB from the Indian subcontinent, 6 of 9 patients (67\%) were diagnosed at surgery, which was considered the treatment of choice [8]. In another retrospective study, mucosal biopsies had a poor yield and surgery was required both for establishing the diagnosis and treatment of GDTB [5]. In contrast, a more recent study from New Delhi documented a high diagnostic yield with endoscopic pinch and snare biopsy for histological diagnosis of GDTB [9]. In the latter study, 12 of 13 pa- 
tients (92.3\%) could be treated successfully with a combination of antituberculous therapy (ATT) and endoscopic dilatation [9].

The present study attempted to evaluate efficacy of nonsurgical management of GDTB using a combination of ATT along with endoscopic balloon dilatation of tuberculous strictures.

\section{Patients and methods}

All consecutive patients diagnosed with GDTB at GB Pant hospital New Delhi from 2009 to 2017 were prospectively evaluated. Of these, a case series 13 cases was published in 2012 which showed significant short-term improvement [9].

Diagnosis of GDTB was made on the basis of demonstration of caseating epithelioid granulomas and/or AFB on ZN stain in duodenal and or gastric biopsies. Patients with gastroduodenal lesions at endoscopy with non-specific histopathological features were also considered to have GDTB, provided they had definitive evidence of TB at any other site.

Patients underwent detailed clinical examination, biochemical investigations, tuberculin testing, and chest x-ray. IGRA, tissue for TB polymerase chain reaction, and culture were not done, as they were costly investigations. The Barium Upper Gastrointestinal Tract series and abdominal CECT (contrast-enhanced computed tomography) were done clinically when indicated. Upper gastrointestinal endoscopy (UGIE) was done under conscious sedation and multiple $(6-8)$ pinch biopsies from the involved mucosa of stomach or duodenum were taken. Endoscopic mucosal resection (EMR)/snare biopsy was done subsequently if the pinch biopsies were inconclusive. Mucosal and EMR biopsies samples were processed for histopathological examination and Ziehl Neelsen (ZN) stain. CECT was done to evaluate the extent of gastroduodenal disease and also to identify lesions elsewhere in the abdomen. Stricture was documented on imaging, (either barium or abdominal CECT) or endoscopically. All patients with a confirmed diagnosis of GDTB were treated with weight-based ATT for a period ranging between 6 and 9 months. Patients who could not tolerate a liquid diet underwent dilatation before starting ATT. Dilatation was also done in patients in whom no significant improvement occurred in 2 to 4 weeks after ATT. Endoscopic balloon dilatation was done in patients with persistent vomiting using the technique described by us earlier [9]. The ATT given was usually four drugs (SHRZ) in the intensive phase and two drugs (HR) in the continuation phase. Intramuscular streptomycin and syrup rifampicin were used initially in patients who were unable to take oral medicines due to recurrent vomiting. The endpoint of endoscopic dilatation was successful dilatation with an $18-\mathrm{mm}$ "through the scope" balloon (Wilson-Cook, United States). On follow-up evaluation, UGIE and barium study was repeated in patients with recurrent vomiting. Patients in whom symptoms persisted despite ATT and endoscopic dilatation were considered to have failure of medical management and were referred for surgery or offered placement of a self-expanding metal stent (SEMS). Post-ATT patients were followed up on an outpatient basis or through telephonic conversation for any clinical recurrence of symptoms in the long term. Eradication of tuberculosis was confirmed by clinical improvement of symptoms and endoscopic healing. Anthropometric measurements (weight and body mass index [BMI]) were recorded before starting treatment and repeated 9 to 12 months after completion of ATT.

\section{Statistical methods}

Mean with $95 \%$ confidence interval and median were calculated for continuous data. The Student's t-test was used for continuous variables to identify the difference between the two groups. $P<0.05$ was considered as significant.

\section{Results}

Fifty-two patient with GDTB were enrolled during the study period from 2009 to 2017. Mean age of the patients was 28.5 years (95\% Cl: 28.4-28.6). There was a significant male predominance as 35 of patients (67\%) were male. Presenting symptoms were vomiting ( $n=51,98 \%)$, weight loss $(n=52,100 \%)$ and fever $(\mathrm{n}=12,23 \%)$. Mean BMI at presentation was $16.5 \mathrm{~kg} / \mathrm{m}^{2}(95 \% \mathrm{Cl}$ : $16.48-16.57)$. Past history of tuberculosis was elicited in eight patients (15\%). Twenty-three patients (44\%) had evidence of tuberculosis elsewhere.

Upper gastrointestinal endoscopy was done in all patients; all but one had a stricture in the stomach, duodenum or at both sites ( $>$ Fig. 1 ). The patient with the non-stricturing disease had a nodular mass in the antrum on UGIE. The site of involvement in GDTB was duodenum ( $n=37,71 \%)$, stomach contiguous with duodenum $(n=13,25 \%)$ or only stomach $(n=2$, $3.8 \%$ ). The first and second part of the duodenum was the most common site of involvement with GDTB in the duodenum (42\%), whereas the pyloric area was the most common site of involvement in the stomach ( $\mathbf{F i g . 2}$ ).

CECT examination provided additional information in 23 patients (44\%). It showed abdominal lymph nodes in 10; pulmonary and pleural in two patients each; isolated jejunal, ileal, coIonic; and a combination of multiple small bowel lesions in one patient each. Five patients have involvement in more than two sites (dissemination).

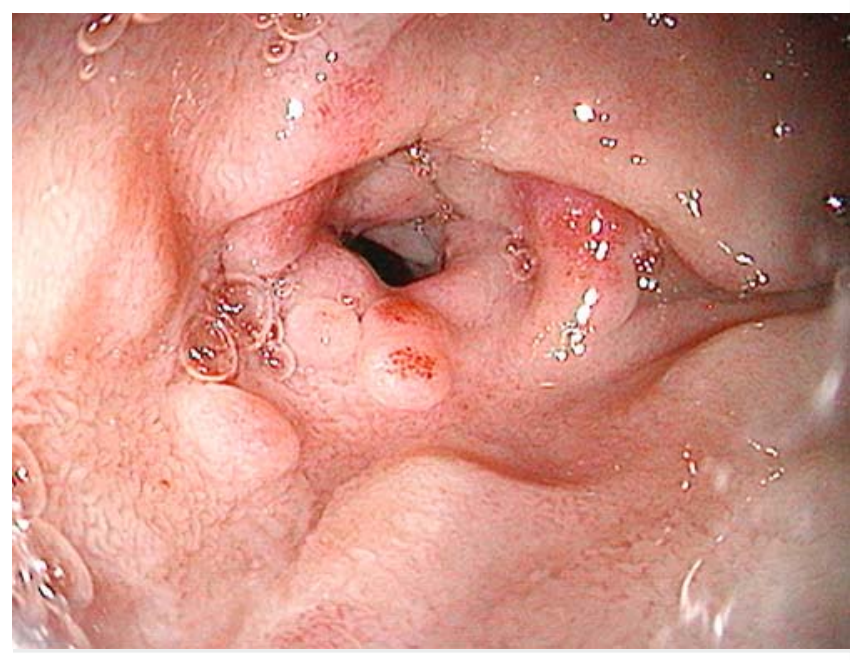

- Fig. 1 Tubercular duodenal stricture. 


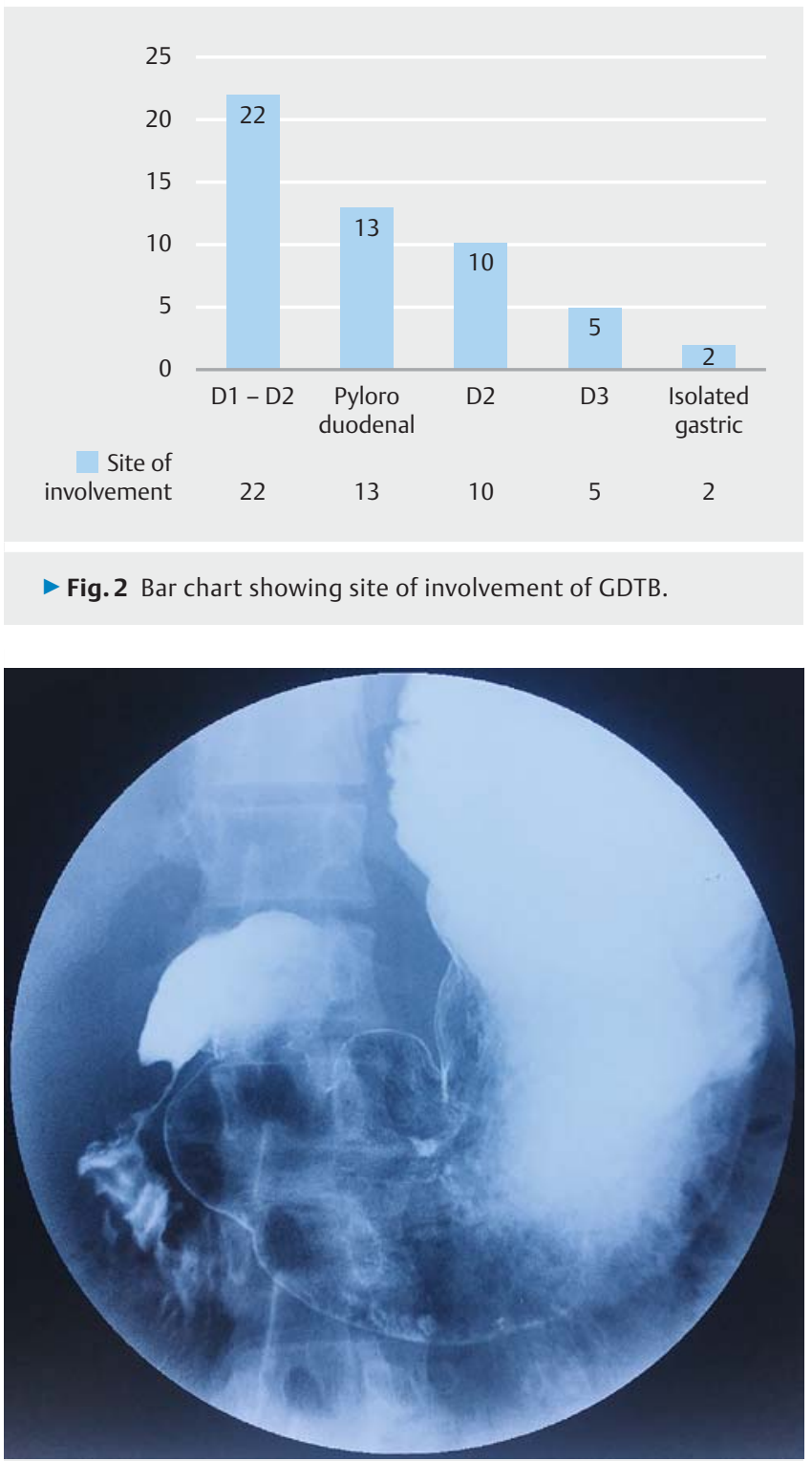

Fig. 3 Barium study showing duodenal stricture.

Each of the 52 patients underwent endoscopy and pinch biopsy of the abnormal-appearing mucosal lesions. In 36 patients (69.2\%) granulomas were seen on histopathological exam (HPE) and acid-fast bacilli (AFB) were seen in three patients (5.7\%). Snare biopsy or EMR was done in 10 patients, seven of whom (70\%) had epithelioid granuloma on HPE and one patient had AFB on ZN staining. Therefore, an accurate histopathological diagnosis could be made in 43 of the 52 patients (83\%).

\section{Management and follow-up}

Forty-two patients required endoscopic balloon dilatation in addition to the ATT drug therapy ( $\triangleright$ Fig. 3 and $\triangleright$ Fig.4). A median of two sessions (range $0-6$ ) were required in these patients. In patients with multiple strictures, dilatation was done for the

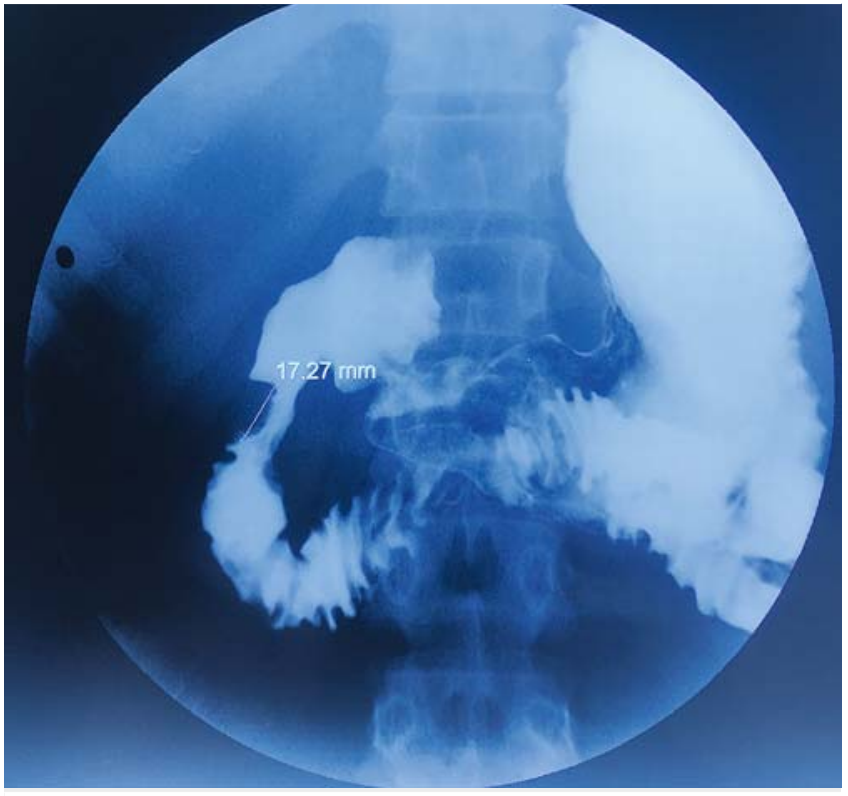

Fig. 4 Duodenal stricture post dilatation.

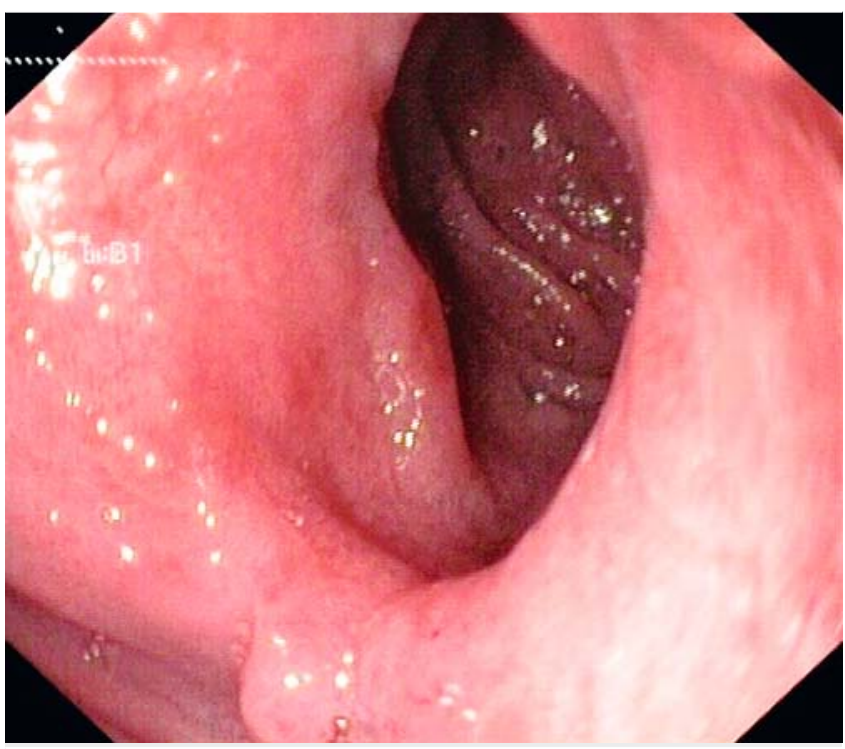

- Fig. 5 Duodenal stricture post-dilatation and completion of ATT.

critical strictures and the rest of the noncritical strictures responded to ATT.

There were two complications post-endoscopic dilatation. One patient had retroperitoneal perforation and was managed conservatively whereas one patient had an intraperitoneal perforation requiring surgery. Endoscopic dilatation failed in four cases: two patients (3.8\%) underwent surgery and one patient underwent placement of a SEMS. One patient who had severe malnutrition preoperatively and underwent a feeding jejunostomy died postoperatively due to sepsis. Patients were able to resume their normal diet usually within 7 days post-endoscopic dilatation. Complete amelioration of vomiting was seen in all responders within a period of 4 to 6 weeks after initiation of de- 
finitive therapy ( $\mathbf{F i g . 5 )}$. Post completion of therapy, mean BMI was $20.5 \mathrm{~kg} / \mathrm{m}^{2}$ which was an increase of $4 \mathrm{~kg} / \mathrm{m}^{2}$ over the baseline value $(P<0.001)$. These patients were followed up prospectively for any recurrence of symptoms both by outpatient visits and telephonic follow-up. The median period of follow up was 23.5 months (IQR 11.5-52 months). None of the patients reported any recurrence of symptoms.

\section{Discussion}

Abdominal tuberculosis has a strong predilection for the lleocecal region, which accounts for more than $50 \%$ of patients with gastrointestinal TB [2]. Tuberculous involvement of the stomach and duodenum are uncommon even in countries where the disease is endemic. In this prospective study, we present our clinical experience of 52 patients with GDTB who were diagnosed at our center over a period of 8 years from 2009 to 2017. The salient features of our study are the relatively large number of patients and the long duration of the study and follow-up period.

The dominant feature of the clinical presentation was presence of recurrent vomiting in 51 (98\%) of 52 patients. This underscores the fact that GDTB presents predominantly with features of gastric outlet obstruction. A recent study has shown that GDTB and corrosive ingestion appear to be almost as common as peptic ulcer disease as etiology of benign gastric outlet obstruction in the Indian subcontinent [10].

The most common site of obstruction was the junction between the first and second part of the duodenum in $42 \%$ of patients followed by the antro-pyloric segment. The duodenal bulb and the second part of the duodenum were more commonly involved than the distal duodenum (third and fourth parts), suggesting a gradient starting from the duodenal bulb which is most vulnerable whereas the third and fourth parts of the duodenum are least vulnerable. The reason for this gradient is currently unknown.

One of the strengths of our study has been demonstration of the high yield of granulomatous pathology on endoscopic biopsy specimens. Previous studies have shown that yield of endoscopic biopsies in establishing a histological diagnosis is less than $10 \%[5,7,11]$. These studies have therefore recommended that surgery is the modality of choice for both diagnosis and treatment of GDTB $[5,11]$. In this prospective study, we have conclusively shown that histologic yield is proportionate to quantity of tissue sent for histopathology. As a part of the study protocol, we were taking six to eight biopsy pieces from the abnormal area. If the report was inconclusive, we followed it up by doing either a snare biopsy or EMR. This is the main reason that we were able to demonstrate either epithelioid granulomas or AFB in more than $80 \%$ of patients. A similar high yield of endoscopic biopsy for demonstration of granulomatous pathology previously was reported by Jain et al albeit the sample size was only seven patients [12].

The current study makes a strong a case for a paradigm shift towards nonsurgical management of GDTB. Surgical management was required in only three of 52 patients with GDTB $(5.8 \%)$ in our study. This is at variance with two earlier reports from India in which the vast majority of patients under- went surgical intervention for both diagnosis and treatment of GDTB $[5,11]$. The efficacy of the nonsurgical approach is underscored by the fact that most of the patients were able to resume their normal diet within a mean of 7 days following endoscopic dilatation. Complete amelioration of vomiting was seen in all patients within a period of 4 to 6 weeks after initiation of definitive therapy. In addition to subjective improvement in symptoms, objective assessment showed an increase in BMI, suggesting a significant improvement in patients' nutritional status. Efficacy of endoscopic balloon dilatation for GDTB has previously been shown in two brief reports $[13,14]$.

\section{Conclusion}

In summary, we have shown that most patients with GDTB present with features of gastric outlet obstruction. Endoscopybased techniques should be considered as the standard operating procedure in diagnosis and management of patients with suspected GDTB. Surgical intervention is required only in the relatively small proportion who do not respond to the combination of ATT and EBD.

\section{Competing interests}

None

References

[1] Rathi P, Gambhire P. Abdominal tuberculosis. J Assoc Physicians India 2016; 64: $38-47$

[2] Bhansali SK. Abdominal tuberculosis. Experiences with 300 cases. Am J Gastroenterol 1977; 67: 324-337

[3] Sharma MP, Bhatia V. Abdominal tuberculosis. Indian J Med Res 2004; 120: 305

[4] Debi U, Ravisankar V, Prasad KK et al. Abdominal tuberculosis of the gastrointestinal tract: Revisited. World J Gastroenterol WJG 2014; 20 : $14831-14840$

[5] Rao YG, Pande GK, Sahni P et al. Gastroduodenal tuberculosis management guidelines, based on a large experience and a review of the literature. Can J Surg 2004; 47: 364

[6] Chavhan GE. Duodenal Tuberculosis: Radiological Features on barium studies and their clinical correlation in 28 cases. J Postgrad Med 2003; 49: 214

[7] Agrawal S, Shetty SV, Bakshi G. Primary hypertrophic tuberculosis of the pyloroduodenal area: report of 2 cases. J Postgrad Med 1999; 45: $10-12$

[8] Ali W, Sikora SS, Banerjee D et al. Gastroduodenal tuberculosis. Aust N ZJ Surg 1993; 63: $466-467$

[9] Puri AS, Sachdeva S, Mittal VV et al. Endoscopic diagnosis, management and outcome of gastroduodenal tuberculosis. Indian J Gastroenterol 2012; 31: 125-129

[10] Maharshi S, Puri AS, Sachdeva S et al. Aetiological spectrum of benign gastric outlet obstruction in India: new trends. Trop Doct 2016; 46: $186-191$

[11] Negi SS, Sachdev AK, Chaudhary A et al. Surgical management of obstructive gastroduodenal tuberculosis. Trop Gastroenterol Off J Dig Dis Found 2003; 24: 39-41 
[12] Jain S, Kumar N, Jain SK. Gastric tuberculosis. Endoscopic cytology as a diagnostic tool. Acta Cytol 2000; 44: 987 - 992

[13] Vij JC, Ramesh GN, Choudhary V et al. Endoscopic balloon dilation of tuberculous duodenal strictures. Gastrointest Endosc 1992; 38: $510-$ 511
[14] Rana SS, Bhasin DK, Chandail VS et al. Endoscopic balloon dilatation without fluoroscopy for treating gastric outlet obstruction because of benign etiologies. Surg Endosc 2011; 25: 1579-1584 